\title{
A Case of Posterolateral Corner Injury with Segond Fracture in Association with Anterior Cruciate Ligament Tear on MRI - A Case Report
}

\author{
Amruta Dinesh Varma ${ }^{1}$, Rajasbala P. Dhande 2 , Suhasini Pattabiraman ${ }^{3}$, Rishabh Gupta ${ }^{4}$, Nagendra Vadlamudi ${ }^{5}$ \\ 1, 2, 3, 4,5 Department of Radiodiagnosis, Jawaharlal Nehru Medical College, Datta \\ Meghe Institute of Medical Sciences, Wardha, Maharashtra, India.
}

\section{INTRODUCTION}

Knee injuries are common in sports and depending on the mechanism of fall, injury of different ligaments can occur. Knee injury mostly involves the anterior cruciate ligament (ACL), and is the most common injury reported on MRI. It can be associated with posterolateral corner (PLC) injury and other osseous injuries, these injuries are rarely reported as they are very complex in structure. A detail knowledge about this complex helps to evaluate the associated ligaments as well. In association with anterior cruciate ligament injuries presence of a Segond fracture indicates occurrence of a concomitant or isolated injury to the posterolateral corner injury. The evaluation of these associated injuries along with anterior cruciate ligament aids in their repair and hence improving the postoperative outcome. Three main components of posterolateral corner are divided into three layers - superficial, middle, and deep. Clinically few tests have been advised to evaluate injury of different structures of knee joint. For example - McMurray's test and Ege's test are used to evaluate meniscus, Lachman test, Anterior drawer test and Pivot Shift Test are performed to evaluate anterior cruciate ligament tear while posterior drawer test is done to evaluate posterior cruciate ligament tear. Availability of special closely coupled extremity coils, high field systems, open system \& extremity units have made MRI supplemental to clinical examination and plain radiographs for virtually all suspected disorders of knee. Here, we are presenting a case of MRI of knee injury involving posterolateral corner injury with anterior cruciate ligament tear and Segond fracture.

Along with anterior cruciate ligament, medial meniscus, other ligaments and osseous injury are associated as well. The other structures responsible for stabilizing the knee joints are posterolateral corner and meniscus, which when involved may need to be treated simultaneously with anterior cruciate ligament tear, for full re stabilization of knee joint. If any associated fracture is present, it has to be treated simultaneously as well.

\section{PRESENTATION OF CASE}

A 27-year-old man came with a history of fall with injury over lateral aspect of left knee joint and swelling, following which he had difficulty in walking. His $\mathrm{x}$-ray report revealed a chip fracture from lateral aspect of lateral condyle of tibia which was suggestive of Segond fracture. Following which MRI was done which showed the full thickness anterior cruciate ligament tear in association with posterolateral corner injury and anterolateral ligament.
Corresponding Author: Dr. Amruta Dinesh Varma, Meghe Heights 2, Flat Number 4, Jawaharlal Nehru Medical College, Datta Meghe Institute of Medical Sciences, Wardha, Maharashtra, India.

E-mail: amrutavarma24@gmail.com

DOI: $10.14260 / j e m d s / 2021 / 513$

How to Cite This Article:

Varma AD, Dhande RP, Pattabiraman $S$, et al. A case of posterolateral corner injury with segond fracture in association with anterior cruciate ligament tear on MRI - a case report. J Evolution Med Dent Sci 2021;10(31):2511-2513, $10.14260 / \mathrm{jemds} / 2021 / 513$

Submission 30-04-2021, Peer Review 01-07-2021, Acceptance 09-07-2021, Published 02-08-2021.

Copyright (C) 2021 Amruta Dinesh Varma et al. This is an open access article distributed under Creative Commons Attribution License [Attribution 4.0 International (CC BY 4.0)] 


\section{DISCUSSION}

In knee injury commonly involved is anterior cruciate ligament of all the ligaments, medial meniscus, however it is associated with other ligament and osseous injury as well. The posterolateral corner is a complex structure with the following components - The upper or the superficial layer consists of the iliotibial band, its anterior extension, and the biceps femoris; the middle layer consists of the lateral patellar retinaculum, two patellofemoral ligaments, and the patellomeniscal ligament and the deep layer consists of lateral capsule, lateral collateral ligament (LCL), coronary ligament, arcuate ligament, popliteus muscle-tendon unit, popliteofibular ligament (PFL), and fabellofibular ligament. ${ }^{1}$ Therefore, to simplify this complex anatomy, here we describe the most sensitive structures of the PLC, with emphasis on three main reinforcements: LCL, popliteus tendon, and PFL. 2,3 The role of the tendons of the PLC muscles and muscles is mainly due to their anatomic relationships and the proximity of their fibular head implants. ${ }^{4}$

The PLC structures are particularly responsible for varus fluctuations also called varus rotations and external tibial rotation. 5 They act as secondary stabilizers, by interacting with the contraction muscles, to avoid internal and posterior translation flexion $\left(0^{\circ}-30^{\circ}\right) .6,7$ Segond fractures are defined as elliptic avulsion fractures of the proximal lateral tibia. They represent the rupture of the lateral capsular fibres of the iliotibial tract or the anterior oblique band of the lateral collateral ligament. ${ }^{8}$ Diagnosis of Segond fractures, means an increased risk of soft tissue injury. ACL injuries allow for excessive internal tibia fractures, and pre-existing and varus angulation is an important component of Segond fracture injury. ${ }^{9}$ MR imaging, which plays a key role in detecting PLC injuries, such as their early detection (i.e., $<3$ weeks can improve outcome.10,11 Other symptoms of back injuries and possible instability after involving fibular ligament injury, popliteus tendon or muscle tears, are haemorrhage and fluid posterior to the popliteus muscle or region of the arcuate ligament and posterolateral capsule. The "arcuate" mark is a nearby fibrous fracture caused by the arousal pressure exerted by the biceps femoris and the fibular collateral ligament, and has a high incidence of injuries associated with the posterolateral capsule and cruciate ligaments. ${ }^{12}$ The following figures 1a, Fig 1b, Fig 2 and an x-ray showing Segond fracture in Fig 3.

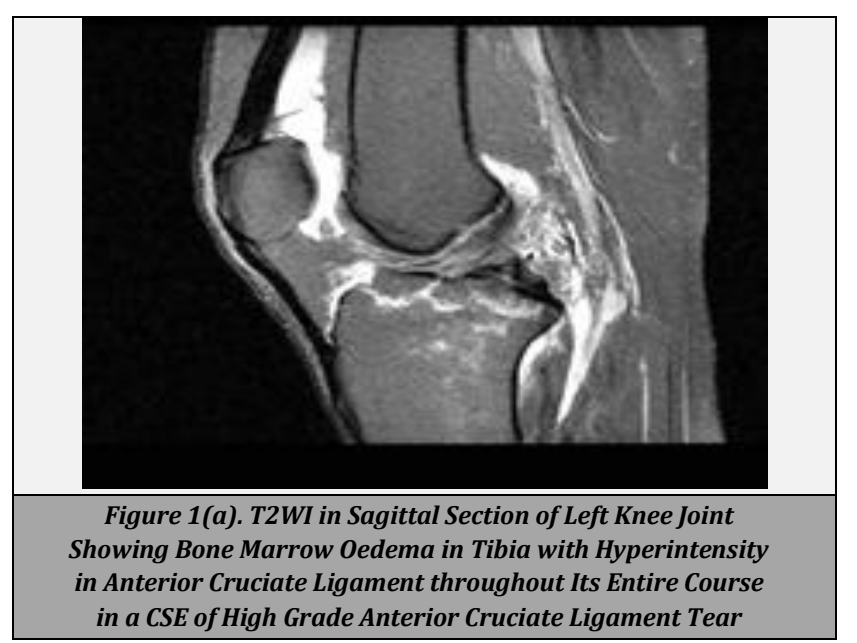

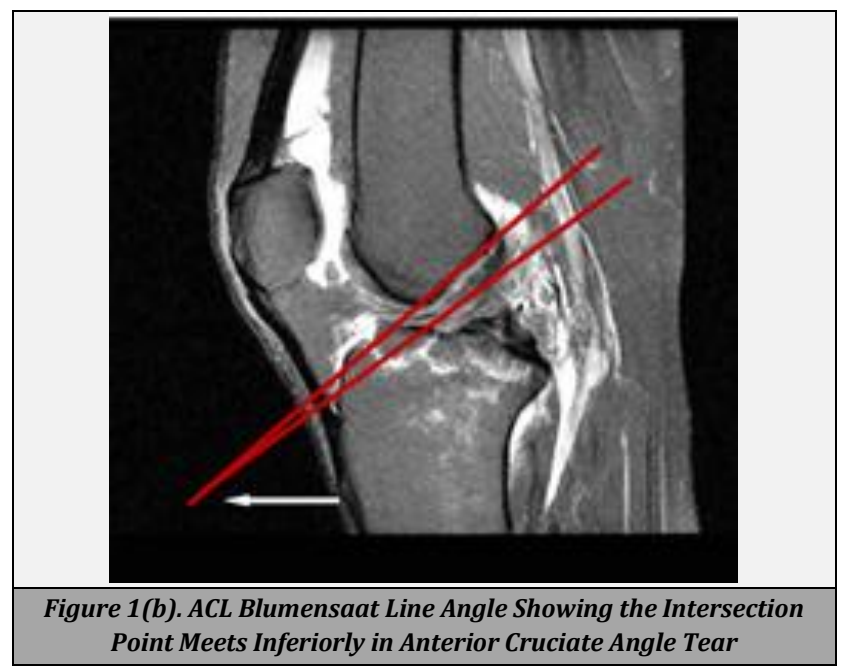
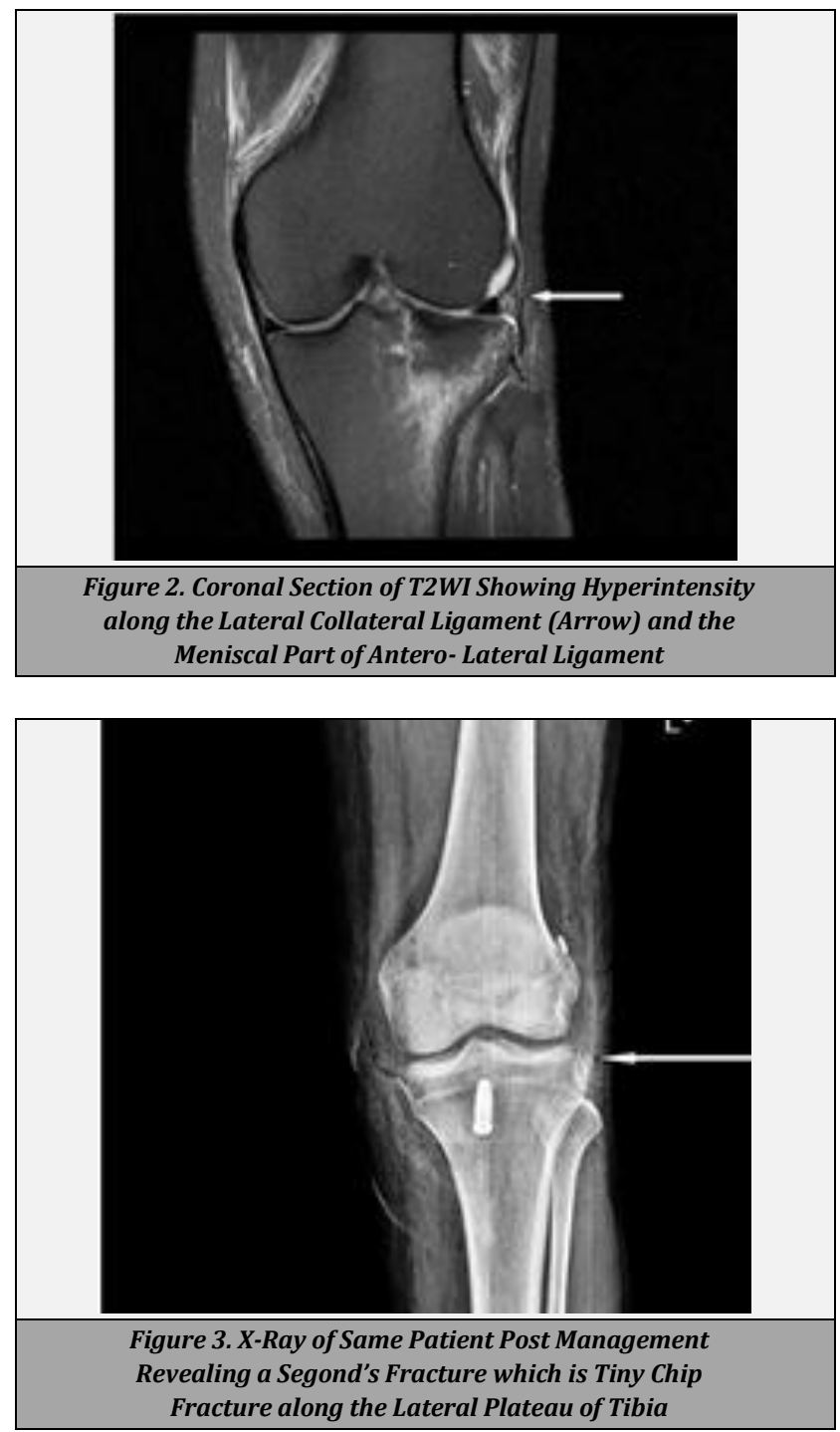

Features of ACL tears: Key features - a) Complete tear - 1 . Depression of the signal strength between parts or female or tibial attachment to T2 WI. 2. A distal or horizontal distal tibial part with a high signal strength near the femoral attachment. 3. Absence of the ligament with effusion and high signal stiffness in the joint space is best seen in T2 WI. 4. Wavy ligament with change in the ACL angle which is formed by line tangent to intercondylar roof and intersecting the line passing through ACL fibres, ideally it should be intersecting superiorly 
while in tear it faces inferiorly. b) Incomplete delicate tears increased signal strength in T2 WI with a thick course and normal course. c) Chronic Tears - 1. Loosening of normal or medium signal strength and thickening or poorly defined path. It can be mistaken for spinal tears, however asymptomatic in the clinic. The typical posterior cruciate ligament is a curved, horizontal structure with low signals in all sequences showing the appearance of a 'hockey stick'. When torn it has a thick central signal that is visible everywhere seen better in the T2 WI. 38 In T2 WI PCL shows the following features:

1. Internal tears: strong thickness, strong signal strength.

2. Eliminate tears: increased signal strength and endless fibres.

3. Particulate tears: flexible stiffness increases signal strength, strong fibres

Financial or other competing interests: None.

Disclosure forms provided by the authors are available with the full text of this article at jemds.com.

\section{REFERENCES}

[1] Seebacher JR, Inglis AE, Marshall JL, et al. The structure of the posterolateral aspect of the knee. J Bone Joint Surg Am 1982;64(4):536-41.

[2] Watanabe $\mathrm{Y}$, Moriya $\mathrm{H}$, Takahashi $\mathrm{K}$, et al. Functional anatomy of the posterolateral structures of the knee. Arthroscopy 1993;9(1):57-62.

[3] LaPrade RF, Ly TV, Wentorf FA, et al. The posterolateral attachments of the knee: a qualitative and quantitative morphologic analysis of the fibular collateral ligament, popliteus tendon, popliteofibular ligament and lateral gastrocnemius tendon. Am J Sports Med 2003;31(6):85460.

[4] Dye SF. An evolutionary perspective of the knee. J Bone Joint Surg Am 1987;69(7):976-83.

[5] Rosas HG. Unraveling the posterolateral corner of the knee. Radiographics 2016;36(6):1776-91.

[6] Nielsen S, Rasmussen 0, Ovesen J, et al. Rotatory instability of cadaver knees after transection of collateral ligaments and capsule. Arch Orthop Trauma Surg 1984;103(3):165-9.

[7] Nielsen S, Ovesen J, Rasmussen 0 . The posterior cruciate ligament and rotatory knee instability: an experimental study. Arch Orthop Trauma Surg 1985;104(1):53-6.

[8] Campos JC, Chung CB, Lektrakul N, et al. Pathogenesis of the segond fracture: anatomic and MR imaging evidence of an iliotibial tract or anterior oblique band avulsion. Radiology 2001;219(2):381-6.

[9] Peltola EK, Mustonen AO, Lindahl J, et al. Segond fracture combined with tibial plateau fracture. Am J Roentgenol 2011;197(6):W1101-4.

[10] Veltri DM, Deng XH, Torzilli PA, et al. The role of the popliteofibular ligament in stability of the human knee: a biomechanical study. Am J Sports Med 1996;24(1):19-27.

[11] Geeslin AG, LaPrade RF. Outcomes of treatment of acute grade-III isolated and combined posterolateral knee injuries: a prospective case series and surgical technique. J Bone Joint Surg Am 2011;93(18):1672-83.

[12] Juhng SK, Lee JK, Choi SS, et al. MR evaluation of the arcuate sign of posterolateral knee instability. AJR Am J Roentgenol 2002;178(3):583-8. 